\title{
Photon shot noise limited detection of terahertz radiation using a quantum capacitance detector
}

\author{
P. M. Echternach, ${ }^{1, \text { a) }}$ K. J. Stone,${ }^{1}$ C. M. Bradford,${ }^{1}$ P. K. Day, ${ }^{1}$ D. W. Wilson, ${ }^{1}$ \\ K. G. Megerian, ${ }^{1}$ N. Llombart, ${ }^{2}$ and J. Bueno ${ }^{3}$ \\ ${ }^{1}$ Jet Propulsion Laboratory, California Institute of Technology, Pasadena, California 91109, USA \\ ${ }^{2}$ Delft University of Technology, Delft, The Netherlands \\ ${ }^{3}$ Space Research Organization of the Netherlands, Utrecht, The Netherlands
}

(Received 7 May 2013; accepted 12 July 2013; published online 1 August 2013)

\begin{abstract}
We observed a sweep rate dependence of the quantum capacitance in a single Cooper-Pair box used as the readout of a Quantum Capacitance Detector. A model was developed that fits the data over five orders of magnitude in sweep rate and optical signal power and provides a natural calibration of the absorbed power. We are thereby able to measure the noise equivalent power of the detector as a function of absorbed power. We find that it is shot-noise-limited in detecting $1.5 \mathrm{THz}$ photons with absorbed power ranging from $1 \times 10^{-22} \mathrm{~W}$ to $1 \times 10^{-17} \mathrm{~W}$. (C) 2013 AIP Publishing LLC.
\end{abstract}

[http://dx.doi.org/10.1063/1.4817585]

A number of approaches for photodetection of sub-mm and far-infrared radiation are being pursued for ground and space based applications. ${ }^{1-6}$ For cooled space based telescopes, requiring photon noise limited detection, the required Noise Equivalent Powers (NEP) are extremely low. As an example, for a background load of $10^{-19} \mathrm{~W}$, the required NEP is of the order of $1 \times 10^{-20} \mathrm{~W} / \mathrm{Hz}^{1 / 2}$ for $1.5 \mathrm{THz}$ radiation. ${ }^{7}$ No detector to date has demonstrated an NEP lower than $0.8 \times 10^{-19} \mathrm{~W} / \mathrm{Hz}^{1 / 2}{ }^{8-10} \mathrm{~A}$ detector based on semiconductor quantum dots has been reported to detect individual photons, but information on photon flux is not available to determine the actual NEP. ${ }^{11}$ We have been developing a concept, the Quantum Capacitance Detector (QCD),${ }^{12-15}$ and in the work described here we demonstrated an NEP of $1.2 \times 10^{-20} \mathrm{~W} / \mathrm{Hz}^{1 / 2}$ at $1.15 \times 10^{-19} \mathrm{~W}$ and photon noise limited performance from $10^{-22} \mathrm{~W}$ through $10^{-17} \mathrm{~W}$. As part of the characterization process, we have developed a detailed balance model of the quasiparticle population in the readout device, the Single Cooper-pair Box (SCB). ${ }^{16}$ This model provided an insight on the mechanism for the so-called quasi-particle poisoning, a persistent problem in the development of quantum bits based on SCBs and other implementations, rendering this work relevant for research in quantum computation. ${ }^{17-21}$ The QCD is based on the $\mathrm{SCB},{ }^{16}$ a superconducting mesoscopic circuit consisting of a superconducting island connected to a lead electrode (or reservoir) via a small $(100 \times 100 \mathrm{~nm}$ typically) tunnel junction, as shown in Fig. 1. Radiation is coupled to the reservoir via the antenna. When a photon is absorbed in the reservoir, Cooper-pairs are broken, and quasiparticles are created above the superconducting gap edge and trapped inside the reservoir by the higher gap niobium plugs. Quasiparticles can then tunnel to the island, thereby changing the capacitance of the device by $\mathrm{C}_{\mathrm{Q}}$, which in turn changes the resonance frequency of the half-wave resonator. This frequency change is sensed by a

\footnotetext{
${ }^{\text {a) }}$ Author to whom correspondence should be addressed. Electronic mail: pierre.m.echternach@jpl.nasa.gov
}

change in the phase of the microwave passing through the feedline. The island can be biased via a gate capacitor. The capacitance of the island consists of a periodic set of peaks of height $C_{Q}=\left(4 E_{C} / E_{J}\right)\left(C_{g}^{2} / C_{\Sigma}\right)$ above a constant level $C_{\text {geom }}$ determined by the dimensions of the junction. Here, $C_{\Sigma}$ is the sum of the junction and gate capacitance, $C_{\mathrm{g}}$ is the gate capacitance, $E_{C}$ is the charging energy $e^{2} / 2 C_{\Sigma}$, and $E_{J}$ the tunnel junction Josephson energy. The peaks arise due to the quantized nature of the Cooper pair charges and are called the quantum capacitance. ${ }^{17}$ When biased at a voltage corresponding to a peak $\left(\mathrm{C}_{\mathrm{g}} \mathrm{V}_{\mathrm{g}}=1 \mathrm{e}, 3 \mathrm{e} \ldots\right)$, assuming a quasiparticle tunnels to the island, the capacitance will drop from $C_{Q}+C_{\text {geom }}$ to $C_{\text {geom }}$. To transform the SCB into a detector, the reservoir is connected to an antenna, which couples radiation in to break Cooper pairs, generating a population $N_{q p}$ of quasiparticles in the reservoir, which is a function of the optical signal power coupled to the reservoir. This establishes a tunneling rate $\Gamma_{\text {in }}$ onto the island proportional to $N_{q p}$, while quasiparticles can tunnel out of the island with a rate $\Gamma_{\text {out }}$, which is approximately independent of $N_{q p}$. The probability of occupation of the island by one quasiparticle is given by $P_{\text {odd }}\left(N_{q p}\right)=\Gamma_{\text {in }} /\left(\Gamma_{\text {in }}+\Gamma_{\text {out }}\right)$. The average value of the capacitance peak will then be given by $C_{\text {peak }}=C_{\text {geom }}+C_{Q}$ $\left(1-P_{\text {odd }}\right)$ and is a function of the optical signal power. In order to read out the capacitance, the island is coupled to a half wave resonator. A capacitance change on the island will cause a resonance frequency shift in the resonator. The resonator is coupled on the opposite end to a microwave feedline, and the transmitted power through the feedline is measured using a conventional in-phase-quadrature (IQ) mixer after amplification by a cold $(4.2 \mathrm{~K})$ low noise amplifier followed by room temperature amplifiers.

The sample used in this work consisted of a $5 \times 5$ array of detectors and is shown in Fig. 1. The resonators for each pixel had a slightly different resonant frequency and were all connected to a single feedline, allowing for simple frequency multiplexing. The audio frequency bias was applied also through the microwave feedline. This eliminated an 

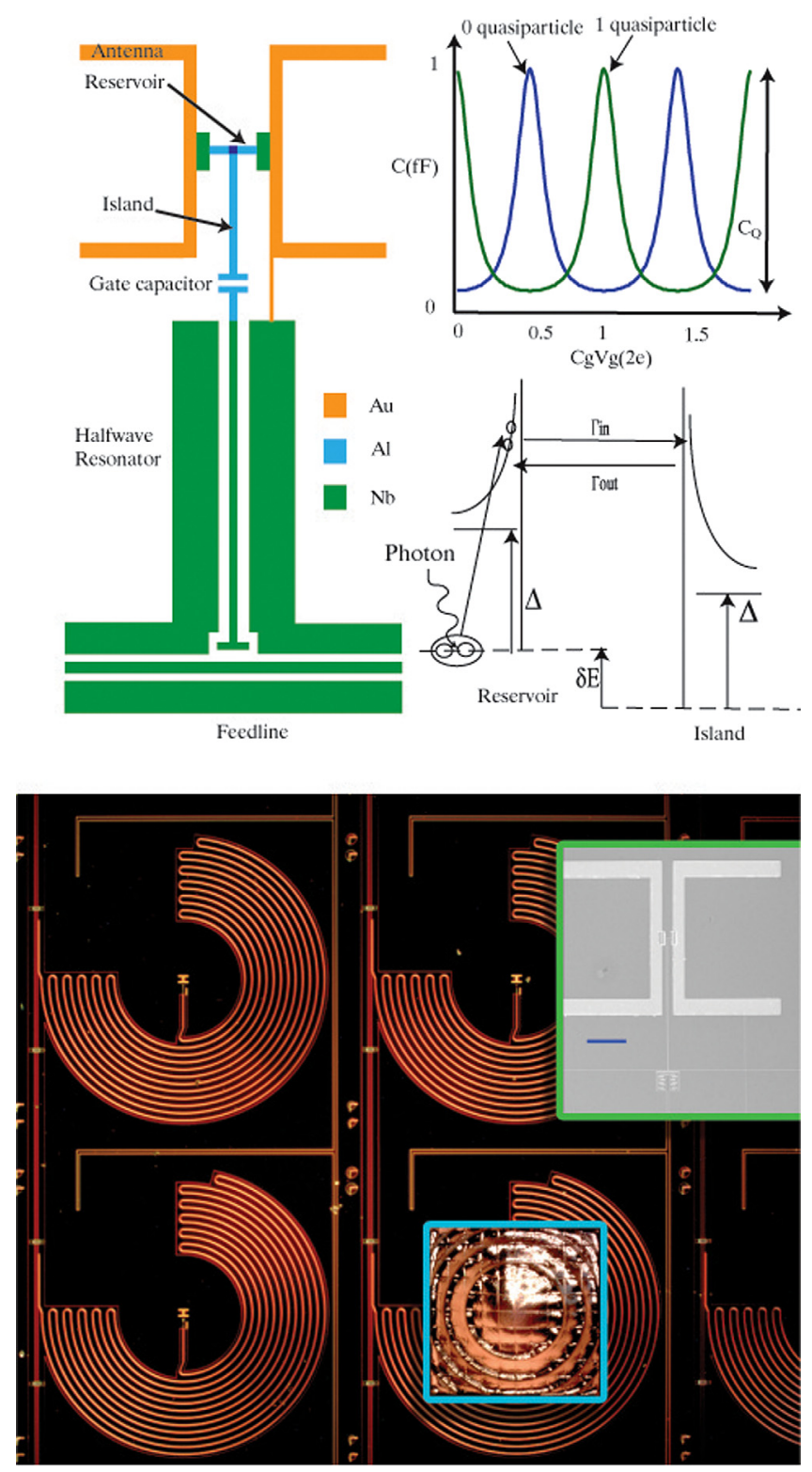

FIG. 1. Top: schematic representation of the Quantum Capacitance Detector. A gold antenna is connected to an aluminum reservoir via two niobium plugs. The reservoir is also connected to an aluminum island via a small $(100 \times 100 \mathrm{~nm})$ tunnel junction and to a ground via a small gold wire stemming out of the antenna. The island is biased via a gate capacitor, which is connected to a half wave resonator. The opposite end of the half wave resonator is capacitively coupled to a microwave feedline. Bottom: four QCD pixels. One element of the Fresnel lens array is shown superimposed on one of the pixels as illustration. The lens array is glued to the back of the detectors which are back side illuminated. A meandering coplanar waveguide (CPW) feedline is capacitively coupled to each CPW half wave resonator (the spiral structures). The double dipole antennas at the opposite end of the resonators are shown in the detail, along with the aluminum reservoir, niobium plugs, gate capacitor and the single Cooper pair box (the long vertical line).

additional gate electrode that would increase $\mathrm{C}_{\text {geom }}$. A gold double dipole antenna is used to couple radiation to an aluminum absorber flanked by niobium plugs used to trap the quasiparticles in the reservoir. In Fig. 1, the island is the vertical line connecting the reservoir to a gate capacitor which couples the device to the open and of the half wave resonator. Terahertz photons are focused onto the detectors by an array of Fresnel lenses glued to the back of the detector wafer. The Fresnel lenses dimensions are optimized for $1.5 \mathrm{THz}$ radiation. The devices are cooled in a dilution refrigerator, and most measurements were performed at $90 \mathrm{mK}$. The optimal temperature for operation, which depends on several device parameters, is discussed elsewhere. ${ }^{12}$ To reduce stray radiation coming from the warmer parts of the dilution refrigerator, the still shield housing the detector, a cylindrical baffle at the base of the interior of the still shield, and a matching baffle mounted directly to the detector housing (which is thermally connected to the mixing chamber) are painted with black epoxy loaded with lamp black carbon powder. Optical illumination is provided by a blackbody source placed outside the still shield but inside the inner vacuum can, in weak thermal contact with the liquid helium bath. The black body source temperature can be varied from 4.2 to $40 \mathrm{~K}$. Black body radiation entered the still shield through a $500 \mu \mathrm{m}$ aperture, a mesh filter with a $10 \%$ band pass around $1.5 \mathrm{THz}$, and a Zytex filter to cut down short wavelength radiation. The optical signal reaching the detector was calculated from the single mode radiation from a black body source using $P_{S}=\int_{0}^{\infty} T(\nu) h \nu / \exp \left(h \nu / k_{B} T\right)-1$, since the detector antenna is sensitive to a single mode. The factor $T(\nu)$ incorporates the measured transmission through the filters and the calculated propagation of the beam through the lens on to the detector, including a calculation of the fraction of the antenna beam pattern filled by the radiation.

We measured the amplitude of the quantum capacitance peaks as a function of gate voltage for a number of illumination power levels, and gate voltage sweep rates as shown in Fig. 2. Published models predict the quantum capacitance is independent of sweep rate or that it should increase with increasing sweep rate. ${ }^{22} \mathrm{We}$ find the quantum capacitance decreases with increasing sweep rates at low levels of

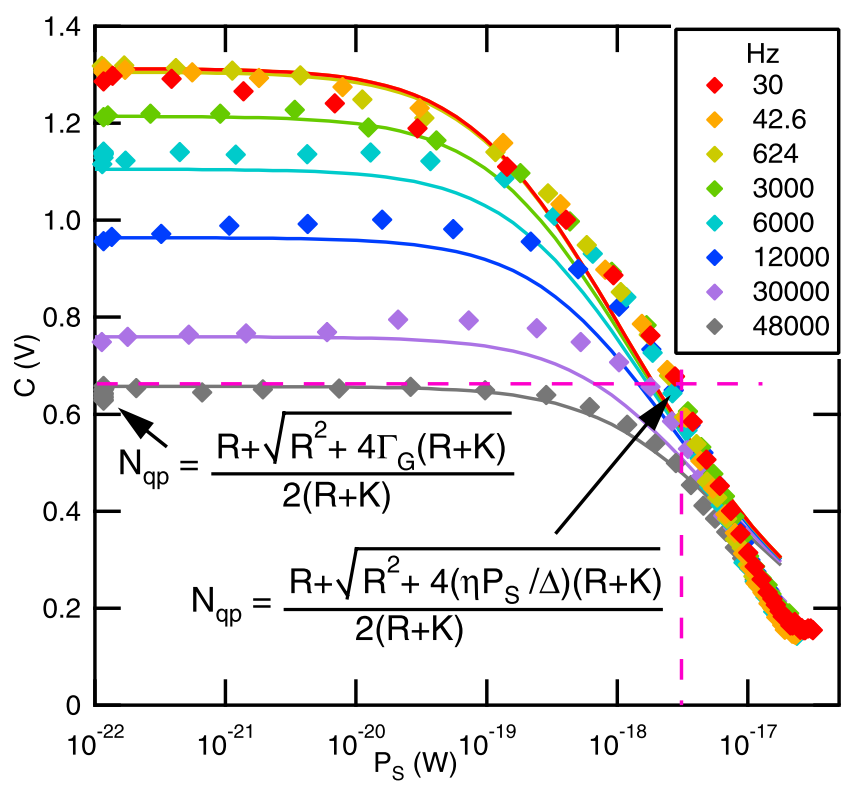

FIG. 2. Sweep rate dependence of optical response. Amplitude of quantum capacitance signal peaks as a function of optical signal power for various gate sweep rates. The dots are experimental values, and solid lines are the fits using the detailed balance model. Since the response is a single valued function of the number of quasiparticles in the reservoir $\mathrm{N}_{\mathrm{qp}}$, by taking a response value at high sweep rates and low signal power and comparing with the same level of response for low sweep rate and high optical signal power, it follows that $\mathrm{Ps}=\Gamma_{\mathrm{G}} \Delta / \eta$. This provides a calibration of the optical signal power absorbed in the reservoir. 
illumination, indicating a higher average number of quasiparticles in the reservoir for higher sweep rates.

To understand this behavior, we developed a simple model depicted schematically in Fig. 3. The energy level of a quasiparticle on the island with respect to the reservoir is represented by their relative position in the figure $(\Delta \mathrm{E})$. At the so-called degeneracy point, the bias voltage at which the quantum capacitance is at a maximum, the energy level of a quasiparticle at the island is lower than at the reservoir, and tunneling onto the island is favored. The tunneling rate into the island is proportional to the density of quasiparticles on the reservoir owing to the dependence of the superconducting gap on the quasiparticle density, ${ }^{12,19}$ so we can write $\Gamma_{i n}=K N_{q p}$. Quasiparticles can elastically tunnel back on to the reservoir from the island with an intrinsic tunneling rate $\Gamma_{\text {out }}{ }^{12,19}$ Quasiparticles on the reservoir recombine with a rate proportional to $N_{q p}\left(N_{q p}-1\right)$ since two quasiparticles are annihilated to create a Cooper Pair and there are $N_{q p}$ $\left(N_{q p}-1\right) / 2$ possible ways of combining $N_{q p}$ quasiparticles. ${ }^{23}$ When the gate voltage is a multiple of $2 e / C_{g}$ the energy level of a quasiparticle on the island is lower than the energy at the reservoir. This will cause the tunneling rate out to be much higher than the tunneling rate at the degeneracy point, and for the sake of simplicity, let us assume an infinite tunneling rate at this point. The gate voltage will change between those two points with a rate equal to twice the gate sweep rate $\Gamma_{G}$. We model the effective tunneling out rate as $\Gamma_{\text {eff }}=\sqrt{\Gamma_{\text {out }}^{2}+\Gamma_{G}^{2}}$. If the intrinsic tunneling rate is much larger than the sweep rate, then the effective tunneling rate is the intrinsic rate. On the other hand, if the intrinsic tunneling rate is much smaller than the sweep rate, the effective tunneling rate is equal to the sweep rate. The detailed balance equation for the number of quasiparticles on the reservoir is

$$
\begin{aligned}
d N_{q p} / d t & =\eta P_{S} / \Delta-R N_{q p}\left(N_{q p}-1\right)-\Gamma_{i n} N_{q p}+\Gamma_{e f f} \\
& =\eta P_{S} / \Delta-(R+K) N_{q p}^{2}+R N_{q p}+\Gamma_{e f f} .
\end{aligned}
$$

Here, $P_{S}$ is the optical signal power, $\Delta$ the superconducting gap of the reservoir, $R$ the quasiparticle recombination rate constant and $\eta$ is the efficiency with which quasiparticles are created by the absorbed photon ( $\eta$ is calculated to be 0.57 for aluminum). ${ }^{24}$ The steady state solution is

$$
N_{q p}=\left(R+\sqrt{R^{2}+4\left(\eta P_{S} / \Delta+\Gamma_{e f f}\right)(R+K)} / 2(R+K) .\right.
$$

The measured response, which is proportional to the amplitude of the quantum capacitance peak at the degeneracy point $\left(C_{\text {peak }}\right)$, will be given by $C=A \Gamma_{\text {out }} /\left(\Gamma_{\text {out }}+\Gamma_{\text {in }}\right)$ $=A /\left(1+K N_{q p} / \Gamma_{\text {out }}\right)$, where $\mathrm{A}$ is a constant dependent on device characteristics and the amplifier chain gain, which we will treat as a fit parameter. The solid lines in Fig. 4 are fits using this equation for the various gate voltage frequencies. This model provides us with a calibration of the signal power absorbed in the reservoir. Since the response is a single valued function of $N_{q p}$, we can take the response at low signal power and high sweep rate and compare it with the same

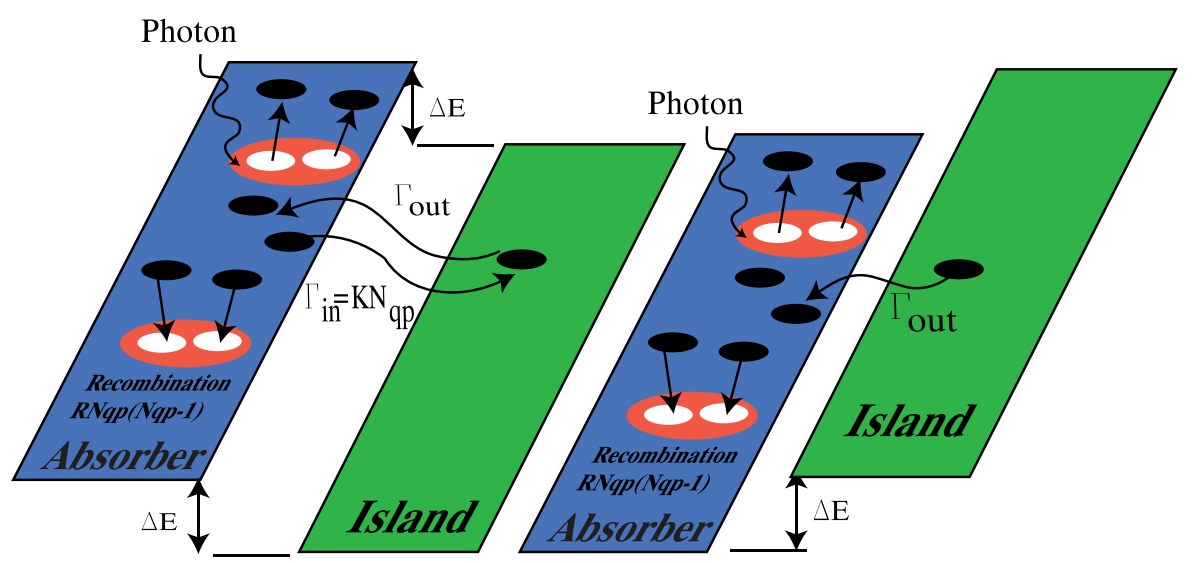

FIG. 3. Detailed balance model of the reservoir quasiparticle population. The relative position of the reservoir and island in the figures corresponds to the quasiparticle energy level at the degeneracy point $(\mathrm{CgVg}=\mathrm{e})$ and at $\mathrm{CgVg}=0,2 \mathrm{e}$. The sources of quasiparticles are Cooper-pair breaking events by absorbed photons, and the island tunneling out rate. The quasiparticle sinks are the recombination rate and the island tunneling in rate. The tunneling out rate will be enhanced at $\mathrm{CgVg}=2 \mathrm{e}$. An effective tunneling out rate is defined as $\Gamma_{e f f}=\sqrt{\Gamma_{\text {out }}^{2}+\Gamma_{G}^{2}}$. 

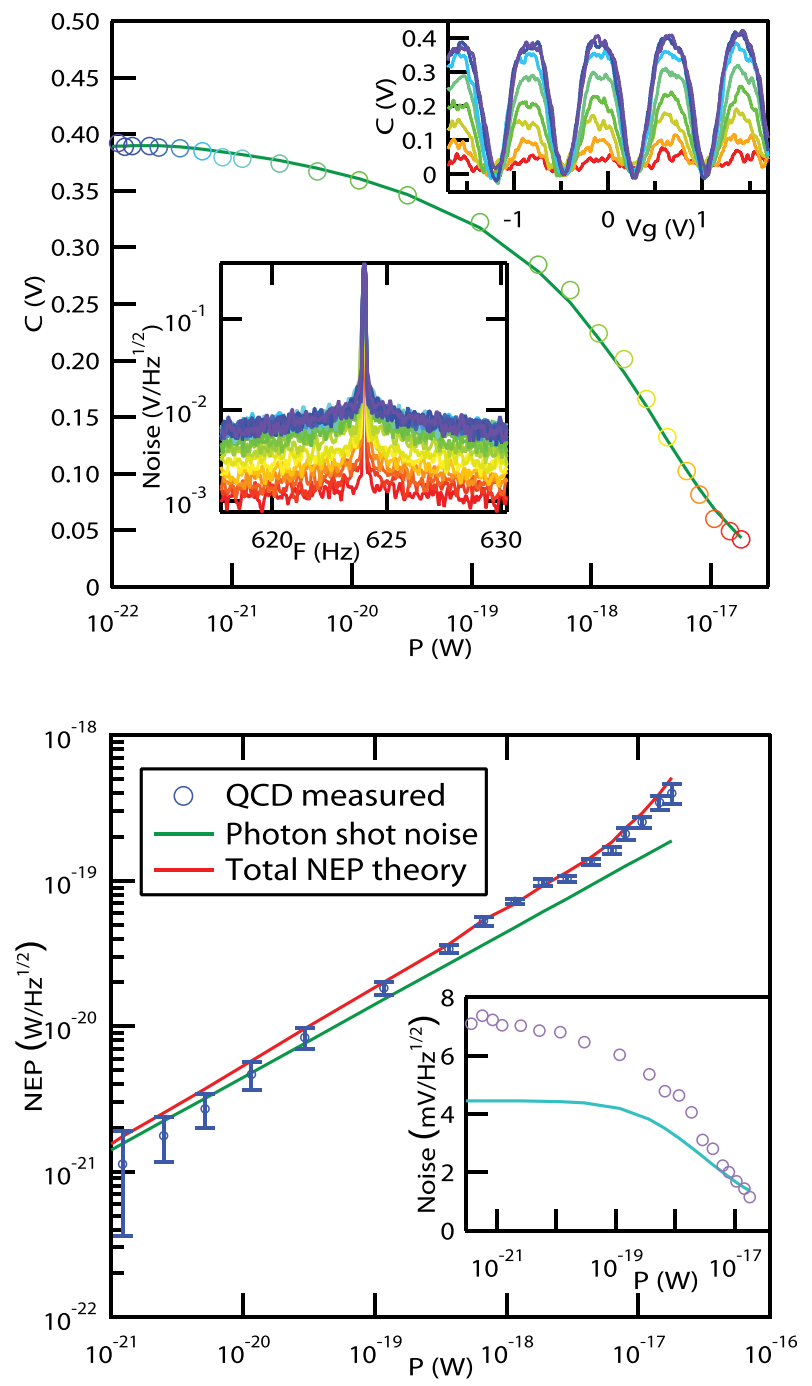

FIG. 4. Top: raw peak-to-peak amplitude (quantum capacitance response) obtained from the spectrum analyzer traces. The solid line is the raw response filtered using a Fourier transform based algorithm. Upper inset: Quantum capacitance traces measured for several optical illuminations using a sweep rate of $624 \mathrm{~Hz}$. Lower inset: raw spectrum analyzer traces for a few optical loadings. Bottom: noise equivalent power calculated from the filtered optical response and the measured noise are plotted as the open blue circles. The photon shot noise of $1.5 \mathrm{THz}$ radiation is plotted as the solid green line. The red line is the calculated NEP taking into account photon shot noise and the shot noise of electron tunneling. Inset: measured noise (circles) compared with the shot noise of electron tunneling (solid line).

level of response for high optical signal power and low sweep rate. Since $N_{q p}$ has to be the same, we arrive at

$$
\begin{aligned}
(R & \left.+\sqrt{R^{2}+4 \Gamma_{G}(R+K)}\right) /(2(R+K)) \\
& =\left(R+\sqrt{R^{2}+4\left(\eta P_{S} / \Delta\right)(R+K)}\right) /(2(R+K))
\end{aligned}
$$

or $\eta P_{S} / \Delta=\Gamma_{G}$. We use this relation to calibrate the $T(\nu)$ term in the black body power calculation.

We measured the quantum capacitance traces for various levels of optical illumination as shown in the upper inset of Fig. 4. We simultaneously use a spectrum analyzer to measure the response and the noise as a function of the calibrated signal power. The spectrum analyzer traces are shown in the inset of Fig. 4 for a few values of optical signal power. The response is the peak-to-peak amplitude obtained from the spectrum analyzer traces. We cross check the peak to peak values obtained from the spectrum analyzer and the quantum capacitance traces. Fig. 4 shows the raw response (blue circles) and the result after filtering the data with a Fourier transform based filter (green solid line). Using the peak-to peak amplitude as the response and using the measured $S(f)$ noise averaged over a range away from the signal peak, we calculated the low frequency noise equivalent power $N E P$ $=S(f) /(d C / d P)$ as a function of optical signal power as shown in Fig. 4. In Fig. 4, we show the photon shot noise $N E P_{p h}=\sqrt{2 h \nu P_{s}}$ as a solid green line. The major source of noise for the QCD is the shot noise of electron tunneling, given by ${ }^{12} \quad S_{s n}(f)=\sqrt{2 A^{2}\left(\Gamma_{\text {in }} \Gamma_{\text {out }} / \Gamma_{\Sigma}\right) /\left(\Gamma_{\Sigma}^{2}+(2 \pi f)^{2}\right)}$. Other noise mechanisms such as phase noise associated with two-level charge fluctuators (TLFs), generationrecombination noise, and Fano noise are much smaller and will not be included here. ${ }^{12}$ The NEP associated with the shot noise of electron tunneling will be given by $N E P_{s n}=S_{s n}(f) /(d C / d P)$. The total expected NEP will then be $N E P_{t o t}=\sqrt{N E P_{p h}^{2}+N E P_{s n}^{2}}$ and is plotted in Fig. 4 as the solid red line, showing very good agreement with the experimental data. The error bars were calculated from $\delta N E P=S(f)\left(\partial^{2} P / \partial C^{2}\right) \delta C$, where $\delta C$ is the standard deviation of the difference between the filtered and the raw response obtained from the spectrum analyzer traces. The inset in Fig. 4 shows the measured noise as a function of optical signal power (blue circles), and the calculated shot noise of electron tunneling using the values of $\mathrm{R}(5500 \mathrm{~Hz}), \mathrm{K}$ $(2000 \mathrm{~Hz})$, and $\Gamma_{\text {out }}(1900 \mathrm{~Hz})$ obtained from the fits as the solid line.

The model developed here is also relevant to the development of quantum bits based on SCBs. The effective tunneling out rate $\Gamma_{\text {eff }}$ acts as a source of quasiparticles for the reservoir, effectively increasing the lifetime of quasiparticles generated by unidentified noise sources. For a device with low intrinsic tunneling out rate $\Gamma_{\text {out }}$, gate sweep rate $\Gamma_{G}$ and a noise source delivering a power $P_{\text {noise }}$ to the reservoir, there will be a residual number of quasiparticles given by $N_{q p}=\left(R+\sqrt{R^{2}+4\left(\left(\eta P_{\text {noise }} / \Delta\right)+\Gamma_{G}\right)(R+K)}\right) / 2(R+K)$.

To minimize the residual population, the sweep rate has to be adjusted to be equal or smaller than the rate of production of quasiparticles by the noise source. In summary, we developed a detailed balance model for the quasiparticle population in the reservoir of a Single Cooper-pair box acting as the readout of a Quantum Capacitance Detector. This model provides a way of calibrating the absorbed power in the reservoir. Using this calibrated power and the measured signal and noise, we demonstrated photon-noise limited operation of the detector at $1.5 \mathrm{THz}$ over a power range spanning five orders of magnitude. The model also provides insight on the mechanism of quasiparticle poisoning in Single Cooper-pair Box based qubits.

We thank Richard Muller for electron beam lithography, Jonathan Kawamura for assistance with optical alignment of the detectors, and Per Delsing for fruitful discussions. The research was carried out at the Jet Propulsion Laboratory, California Institute of Technology, under a contract with the National Aeronautics and Space Administration. 
${ }^{1}$ C. Hunt, J. Bock, P. Day, A. Goldin, A. Lange, H. LeDuc, A. Vayonakis, and J. Zmuidzinas, Proc. SPIE 4855, 318 (2003).

${ }^{2}$ P. K. Day, H. G. LeDuc, B. A. Mazin, and A. Vayonakis, Nature (London) 425, 817 (2003).

${ }^{3}$ J. Wei, D. Olaya, B. Karasik, S. V. Pereverzev, A. V. Sergeev, and M. E. Gershenson, Nat. Nanotechnol. 3, 496 (2008).

${ }^{4}$ P. Khosropanah, R. A. Hijmering, M. Ridder, M. A. Lindeman, L. Gottardi, M. Bruijn, J. van der Kuur, P. A. J. de Korte, J. R. Gao, and H. Hoevers, AIP Conf. Proc. 1185, 42 (2009).

${ }^{5}$ D. Morozov, P. D. Mauskopf, P. Ade, M. Bruijn, P. A. J. de Korte, H. Hoevers, M. Ridder, P. Khosropanah, B. Dirks, and J.-R. Gao, AIP Conf. Proc. 1185, 48 (2009).

${ }^{6}$ A. D. Beyer, M. E. Kenyon, P. M. Echternach, B.-H. Eom, J. Bueno, P. K. Day, J. J. Bock, and C. M. Bradford, Proc. SPIE 7741, 774121 (2010).

${ }^{7}$ C. M. Bradford, M. E. Kenyon, W. Holmes, J. Bock, and T. Koch, Proc. SPIE 7020, 702010 (2008).

${ }^{8}$ B. S. Karasik and R. Cantor, Appl. Phys. Lett. 98, 193503 (2011).

${ }^{9}$ A. D. Beyer, P. M. Echternach, M. E. Kenyon, M. C. Runyan, B. Bumble, C. M. Braford, J. J. Bock, and W. A. Holmes, IEEE. Trans. Appl. Supercond. 23, 2100104 (2013).

${ }^{10}$ S. J. C. Yates, J. J. A. Baselmans, A. Endo, R. M. J. Janssen, L. Ferrari, P. Diener, and A. M. Baryshev, Appl. Phys. Lett. 99, 073505 (2011).

${ }^{11}$ S. Komiyama, O. Astafief, V. Antonov, T. Kutsuwa, and H. Hirai, Nature 403, 405 (2000).
${ }^{12}$ M. D. Shaw, J. Bueno, P. K. Day, C. M. Bradford, and P. M. Echternach, Phys. Rev. B 79, 144511 (2009).

${ }^{13}$ J. Bueno, M. D. Shaw, P. K. Day, and P. M. Echternach, Appl. Phys. Lett. 96, 103503 (2010).

${ }^{14}$ J. Bueno, N. Llombart, P. K. Day, and P. M. Echternach, Appl. Phys. Lett. 99, 173503 (2011).

${ }^{15}$ K. J. Stone, K. G. Megerian, P. K. Day, P. M. Echternach, J. Bueno, and N. Llombart, Appl. Phys. Lett. 100, 263509 (2012).

${ }^{16}$ V. Bouchiat, D. Vion, P. Joyez, D. Esteve, and M. H. Devoret, Phys. Scr. T76, 165 (1998).

${ }^{17}$ T. Duty, G. Johansson, K. Bladh, D. Gunnarsson, C. Wilson, and P. Delsing, Phys. Rev. Lett. 95, 206807 (2005).

${ }^{18}$ B. S. Palmer, C. C. Sanchez, A. Naik, M. A. Manheimer, J. F. Schneiderman, P. M. Echternach, and F. C. Wellstood, Phys. Rev. B 76, 054501 (2007).

${ }^{19}$ M. D. Shaw, R. M. Lutchyn, P. Delsing, and P. M. Echternach, Phys. Rev. B 78, 024503 (2008).

${ }^{20}$ J. M. Martinis, M. Ansmann, and J. Aumentado, Phys. Rev. Lett. 103, 097002 (2009).

${ }^{21}$ G. Catelani, J. Koch, L. Frunzio, R. J. Schoelkopf, M. H. Devoret, and L. I. Glazman, Phys. Rev. Lett. 106, 077002 (2011).

${ }^{22}$ J. F. Schneiderman, P. Delsing, G. Johansson, M. D. Shaw, H. M. Bozler, and P. M. Echternach, AIP Conf. Proc. 850, 931 (2006).

${ }^{23}$ C. M. Wilson and D. Prober, Phys. Rev. B 69, 094524 (2004).

${ }^{24}$ A. G. Kozorezov, A. F. Volkov, J. K. Wigmore, A. Peacock, A. Poelaert, and R. den Hartog, Phys. Rev. B 61, 11807 (2000). 